\title{
BETRUG
}

\section{Bevor der Staatsanwalt klingelt}

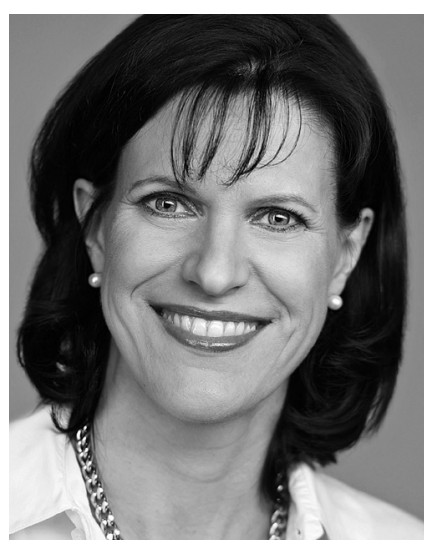

VON BIRGIT GALLEY

Birgit Galley studierte Betriebswirtschaft an der HumboldtUniversität Berlin mit dem Schwerpunkt Recht und ist seit Anfang der 1990er Jahre auf dem Gebiet der Korruptionsprüfung tätig. Als Geschäftsführerin der Forensic Management $\mathrm{GmbH}$ betreut sie seit Mitte der 199oer Jahre Unternehmen verschiedener Branchen sowohl in der repressiven Aufbereitung eingetretener Schadensfälle als auch in der präventiven Beratung zum Aufbau geeigneter ComplianceStrukturen. In ihrer Funktion als Direktorin der School GRC ist sie verantwortlich für die Ausbildungen zum MBA, zum Certified Compliance Expert (CCE), zum Certified Investigation Expert (CIE) sowie zum Master of Arts Criminal Investigation (MACl). www.school-grc.de

\author{
Vor Untreue und Betrug sind auch Wohlfahrtsverbände \\ und Sozialunternehmen nicht gefeit. Auch wenn es \\ sich oft um "Verfehlungen Einzelner" handeln sollte, \\ müssen sich Führungskräfte doch rechtzeitig mit \\ Klima und Strukturen in der eigenen Organisation \\ befassen, die solches Fehlverhalten ermöglichen.
}

Warum sind wir so besonders empört und betroffen, wenn in den Medien über Betrugs- oder Untreuefälle in Sozialunternehmen berichtet wird? Das mag daran liegen, dass es schwer vorstellbar ist, dass sich Menschen, die sich einem belastenden Arbeitsalltag verschrieben haben, irgendwann unredlich verhalten. Dies mag auch daran liegen, dass unser Urvertrauen in ein System, das überwiegend von Vertrauen und Herzenswärme geprägt ist, erschüttert wird.

Das Argument greift um sich, es handele sich dabei - immer wieder - um Verfehlungen eines Einzelnen unter ganz besonderen Umständen, die nicht auf andere Sozialunternehmen übertragbar seien. Das mag stimmen - jedoch nicht so ganz! Wiegt ein Einzelfall einer Verfehlung in einem System, das durch Vertrauen und Mitmenschlichkeit ganz besonders geprägt ist, nicht um ein Vielfaches schwerer? Und fällt es nicht doch auf alle zurück?

\section{Weil wir es uns nicht vorstellen können}

Wirtschaftsdeviantes Verhalten beginnt im Kopf und endet - im großen oder im kleinen Stil - bei Falschabrechnungen oder persönlichen Bereicherungen. Das ist in Sozialunternehmen, Pflegeeinrichtungen oder Wohlfahrtsverbänden nicht anders als in Wirtschaftsunternehmen oder Behörden. Sowohl dem »Beginn im Kopf « als auch dem "Griff in die Kasse» etwas entgegenzusetzen, ist Aufgabe aller
Führungskräfte - egal ob in Unternehmen der Sozial- oder der freien Wirtschaft.

Täterverhalten beginnt nicht spontan. Viele Jahre Erfahrung in der Ermittlerpraxis zeigen, dass Täter - geplant oder nicht - auch einmal klein angefangen haben. Sie entwickeln sich besonders gedeihlich in einem System von falsch verstandenem Vertrauen, vermiedenen oder unzureichenden Kontrollen und ungeklärten Zuständigkeiten.

Nur weil es in dem eigenen Verantwortungsbereich unvorstellbar scheint, dass ein lang bekannter Mitarbeiter oder eine angesehene Führungskraft den Verlockungen des Geldes oder der Macht erliegen könnte, kann dies nicht bedeuten, die Augen davor zu verschließen.

Die Spitze des Eisberges überrascht immer dann, wenn das gesamte Ausmaß einer langen Bereicherungskarriere ersichtlich wird. Aus einem »Einzelfall « - schmerzhaft - zu lernen heißt auch, die nächsten Einzelfälle zu verhindern.

\section{Was die Guten erwarten dürfen}

Gerade bei Betrugs- und Untreuefällen im Sozialbereich geht es nicht immer nur um Geld oder Macht, sondern ganz besonders um Menschen, die in Notsituationen falsch versorgt werden. Sich diesen Menschen verpflichtet zu fühlen und sich für eine Verbesserung ihrer Lebenssituation einzusetzen, ist eine der ehrenvollsten Aufgaben, die wir in einer sozialen Marktwirtschaft finden können. Den hier tätigen Mitarbeitern gebührt nicht 
nur großer Respekt. Sozialunternehmen müssen dem Anspruch dieser Menschen umso mehr gerecht werden, indem sie nicht zulassen, dass deren Arbeit durch das unlautere Tun Einzelner verunglimpft wird und unter einem Generalverdacht von Bereicherungsabsichten Einzelner erstickt. Daher liegt es nicht nur im Verantwortungsbereich, sondern naturgemäß auch im ureigenen Interesse von Sozialunternehmen, die Guten zu schützen und einen Bösen rechtzeitig zu finden.

\section{Was die Bösen erwarten dürfen}

Vermögensschädigendes Verhalten bleibt nie ganz verborgen. Ob Gebühren abgerechnet werden, deren Leistung nicht erbracht wurde, ob Aufwendungen berechnet werden, die für andere Zwecke genutzt werden, oder ob die Betreuung von Personen nachgewiesen wird, die es in dieser Zahl gar nicht gab ... Unendlich viele Szenarien - und auch Gründe - in einem auf Vertrauen fußenden System sind denkbar, um aus einer kreativen Gestaltung ein Vermögensdelikt wachsen zu lassen. Dies schafft auch immer Beobachter und Mitwisser.

Transparenz über Leistungserbringung, Abrechnungsmodi und Verantwortlichkeiten sind der natürliche Feind von wirtschaftsdevianten Machenschaften. Diese Transparenz ist jedoch auch nur dann sinnvoll, wenn Kontrollgremien willens und in der Lage sind, Kontrollen und Aufklärung voranzubringen, schädigendes Verhalten zu erkennen, Sanktionen konsequent durchzusetzen und somit verlorenes Vertrauen wieder zurückzugeben.

Damit steigt nicht nur die Entdeckungswahrscheinlichkeit eines potenziellen Täters, sondern auch sein wahrgenommenes Risiko, dass sein Verhalten sanktioniert wird. Konsequenterweise muss er dann nicht nur die Früchte dieses Tuns verlieren, sondern auch den Zugang zu dem Baum, an dem weitere Früchte hängen.

\section{Was Sozialunternehmen tun können}

Den Dachorganisationen der einzelnen Sozialunternehmen kommt eine weitaus größere Bedeutung als bislang zu. Eine vertrauenswürdige Anlaufstelle für Beobachter unlauterer Verhaltensweisen ist ein zentrales Instrument, um frühzeitig schä- digendes Verhalten zu entdecken. Solange diese Meldungen auf fachliche Kompetenz, umfassende Ermittlungserfahrung, neutrale Sachverhaltsermittlung und professionelle Verschwiegenheit stoßen, wird mit einer solchen Vertrauensstelle auch kein Boden für Falschmeldungen oder Denunziantentum bereitet.

Aus Kapazitäts- und organisatorischen Gründen wird nicht jedes Sozialunternehmen solche Anlaufstellen schaffen können. Die Dachorganisationen sollten solche Vertrauenspersonen installieren, die die erlangten Hinweise entweder an die betroffenen Mitgliedsunternehmen zur Überprüfung oder an eine fest definierte neutrale und kompetente Kontrollinstanz weiterleiten.

Die Wirtschaftsunternehmen leben derartige Hinweisgebermöglichkeiten bislang in geübter Praxis vor und haben in den vergangenen Jahren umfassende - und fast ausnahmslos positive - Erfahrungen gesammelt.

Es ist nicht zu empfehlen, Hinweise unmittelbar an die Staatsanwaltschaft zur Überprüfung weiterzuleiten. Schließlich ist nicht zu verlangen, dass ein Hinweisgeber vor seiner Meldung als Ermittler tätig wird, daher sind die Hinweise im Informationsgehalt oft noch nicht in der Qualität, dass die Staatsanwaltschaft nach dem Legalitätsprinzip eigene Ermittlung aufnimmt. Zudem ist es auch nicht Aufgabe der Staatsanwaltschaft, jeden Hinweis auf Missstände zu validieren. Diese Aufgabe obliegt den Unternehmen selbst; oft handelt es sich auch nicht um rein strafrechtliche Sachverhalte, sondern vielfach um arbeits- oder zivilrechtliche Vergehen. Die Erwartungshaltung an die Staatsanwaltschaft als ausgelagerte Kontrollinstanz der Sozialunternehmen wäre daher unangemessen und ist schlichtweg nicht erfüllbar.

In Erwartung der Neufassung des \$ 612a BGB (1) sind deutsche Unternehmen - und damit auch Sozialunternehmen - gut beraten, für die Kanalisierung von Hinweisen an eine eigene eingerichtete Stellen zu sorgen. Denn die Neufassung des Gesetzes geht davon aus, dass ein Mitarbeiter seine Hinweise dann an die Staatsanwaltschaft geben kann, wenn die Meldung für ihn im Unternehmen nicht zumutbar ist oder seine erfolgte Meldung reaktionslos blieb. (2)

Um also noch vor dem Staatsanwalt von Missständen im eigenen Unternehmen zu wissen und eine Chance auf ei- nen weniger schmerzhaften Lernprozess zu haben, aber auch, um den Erwartungen der Guten gerecht zu werden, muss die Verantwortung bei den Unternehmen selbst bleiben - und damit genau dort, wo sie hingehört!

Sozialunternehmen und ihren Dachorganisationen obliegt nicht zuletzt aufgrund ihrer besonderen Stellung um Vertrauen und menschliches Miteinander eine ganz besondere Verantwortung, Missstände in ihren Unternehmen - und damit auch Betrugs- und Untreuefälle - frühzeitig zu entdecken und wirkungsvoll zu bekämpfen. Wie bei allen Kontroll- und Präventionskonzepten erfordert eine solche Strategie ein vernünftiges Konzept, das

- durch erforderliche Transparenz und ein passendes Regelwerk Vertrauen in die Unternehmensorganisation schafft,

- die richtigen Signale vom Management (3) sendet,

- kompetente und sachorientierte Kontrollinstanzen installiert,

- angemessen Hinweise auf Missstände entgegennehmen kann,

- bereit und fähig zu Sanktionen ist und somit

- die redlichen vor den unredlichen Mitarbeitern schützt.

Dies ist nicht zuletzt auch innerhalb der Sozialunternehmen erforderlich, bevor der Staatsanwalt klingelt und schützt sie potenziell auch davor, dass er es jemals tut.

\section{Anmerkungen}

(1) Auf Bundesebene ist über diese Gesetzesänderung aufgrund unterschiedlicher Rechtsauffassungen und letztlich noch ungeklärter Regelungen bislang nicht abschließend entschieden worden, die SPDFraktion im Deutschen Bundestag plädiert auf ein eigenes Hinweisgebergesetz, auch darüber ist noch nicht befunden worden.

(2) Im Beamtenstatusgesetz ist dies bereits seit 2009 geregelt, für international börsennotierte Unternehmen gilt der vom US-amerikanischen Kongress 2002 verabschiedete Sarbanes-Oxley-Act, der u. a. genau solche Anlaufstellen regelt.

(3) Auch als »Tone from the top« bezeichnet. 